\title{
Article
}

\section{Imatinib Sets Pericyte Mosaic in the Retina}

\author{
Tamás Kovács-Öller 1,2,3,5, , Elena Ivanova 3 ${ }^{3}$ Gergely Szarka 1,2, Ádám J Tengölics 1,2,4, Béla Völgyi \\ 1,2,4.5 and Botir T Sagdullaev ${ }^{3}$ \\ ${ }^{1}$ János Szentágothai Research Centre, University of Pécs, 7624, Pécs, Hungary; kovacstx@gmail.com (T.KÖ.); \\ gergely.sz@gmail.com (G.S.); tengo.adam2@gmail.com (A.T.); volgyi01@gamma.ttk.pte.hu (B.V.) \\ 2 Retinal Electrical Synapses Research Group, National Brain Research Program (NAP 2.0), Hungarian \\ Academy of Sciences, 1051, Budapest, Hungary \\ ${ }^{3}$ Burke Neurological Institute, Department of Ophthalmology, Weill Cornell Medicine, White Plains, New \\ York, USA; eli3001@med.cornell.edu (E.I.); bos2005@med.cornell.edu (B.S.) \\ ${ }_{4}^{4}$ Department of Experimental Zoology and Neurobiology, University of Pécs, 7624, Pécs, Hungary \\ ${ }^{5}$ Medical School, University of Pécs, 7624 Pécs, Hungary \\ * Correspondence: T.KÖ.; kovacstx@gmail.com
}

\begin{abstract}
The nervous system demands an adequate oxygen and metabolite exchange, making pericytes (PCs), the only vasoactive cells on the capillaries, essential to neural function. Loss of PCs is a hallmark of multiple diseases, including diabetes, Alzheimer's, ALS and Parkinson's. Platelet-Derived Growth Factor Receptors (PDGFRs) have been shown to be critical to the PC function and survival. However, how PDGFR-mediated PC activity affects vascular homeostasis is not fully understood. Here, we tested the hypothesis that Imatinib, a chemotherapeutic agent and a potent PDGFR inhibitor, alters the PC distribution and thus induces vascular atrophy. We performed a morphometric analysis of the vascular elements in sham control and Imatinib-treated NG2-DsRed mice. Vascular morphology and the integrity of the blood-retina barrier (BRB) were evaluated using blood albumin labeling. We found that Imatinib decreased the number of PCs and blood vessel (BV) coverage in all retinal vascular layers, this was accompanied by a shrinkage of BV diameters. Surprisingly, the total length of capillaries was not altered, suggesting a preferential effect of Imatinib on PCs. Furthermore, the blood-retina barrier disruption was not evident. In conclusion, our data suggests that Imatinib could help in treating neurovascular diseases and serve as a model for pericyte loss, without BRB disruption.
\end{abstract}

Keywords: pericyte; retina; Imatinib; Gleevec, PDGFR, neurovascular

\section{Introduction}

The Platelet-Derived Growth Factor Receptor-Beta (PDGFR- $\beta$ ) is amongst the major markers expressed by pericytes (PCs) found on the capillaries and responsible for metabolism and oxygen supply. Together with the PDGFR-BB ligand, it promotes vascular maturation and stabilization by recruiting pericytes and their progenitors $[1,2]$.

Although their role has been controversial for a long time [3], it is now clear that PCs are able to regulate blood flow in the nervous tissue as part of the neurovascular unit (Fig. 1c) [4-8]. They express a-SMA, making them capable of active vasoconstriction [9], functionally shown by Ivanova et al. 2017 [6] before. They also have a crucial role in many diseases, such as diabetic retinopathy [6, 10, 11]. In pericyte-deficient mice, hypoxia was reported in the hippocampus, as well as inflammation, and learning and memory impairment [12]. Embryonal ablation of either PDGF-BB or PDGFR- $\beta$ induces microvascular leakage and hemorrhage and is therefore lethal [13, 14]. Interestingly, however, they are only essential in the formation of new blood vessels $[15,16]$, but not in adult blood-retina barrier (BRB) function in the retina [17]. Pericytes regulate BBB in development $[17,18]$ while in the adult CNS, pericyte-deficient blood vessels become dilated without BBB impairment $[19,20]$. 
PCs express PDGFR- $\beta$, endothelial cells (EC) secrete PDGF-BB, which is an important relationship to recruit PCs to blood vessels during development [21]. PDGFR- $\beta$ is present on PCs, whereas mature ECs express PECAM1 (CD31) and both labels are restricted to the vasculature. In addition, PCs and smooth muscle cells (SMCs) express neural-glial marker-2 (NG2, or Chondroitin sulfate proteoglycan 4 (Fig. 1a-b). NG2 is a more suitable marker for pericyte morphological assessment and counting, as the somata of the cells are evenly visible [6, 7, 22] (Fig. 1a, 2).

Imatinib has been potentially used as selective cancer treatment acting through multiple signaling pathways [23-25]. It is a BCR-ABL tyrosine kinase inhibitor and platelet-derived growth factor receptor (PDGFR) and KIT inhibitor, thus induces apoptosis by inhibiting PDGFs but not insulin-induced PI 3-kinase/Akt survival signaling [26]. Imatinib has also been shown to impair the migration of pericytes and fibroblasts in vitro [27].

Pharmacologic intervention with PDGFR- $\beta$ inhibitors, such as Imatinib, has shown significant effects in attenuating angiogenesis by reducing pericyte density. In addition, Imatinib has been shown to successfully inhibit retinal neovascularization in oxygen-induced retinopathy in development by reducing the number of vascular cells, including pericytes [24, 28]. Contrary, targeting PDGFR by Imatinib administration in the adult retina has not been extensively evaluated as a potential model for pathological pericyte loss. Here we show the decrease of vascular areal densities, possibly as a result of PC depletion following either local or systemic treatment of Imatinib in the adult mouse retina.

In most previous studies Imatinib was injected intraperitoneally, ignoring the possible adverse effects of systemic treatment [29] (Fig. 1d). Although, such adverse effects directly linking pericyte depletion to a decrease in BV density and retinal degeneration have not been proven [30, 31]. In summary, inhibition of PDGFs/PDGFRs might be a promising method for aimed PC elimination without BBB disruption. Our findings also suggest that local Imatinib administration could be considered as an efficient treatment modality. 
a

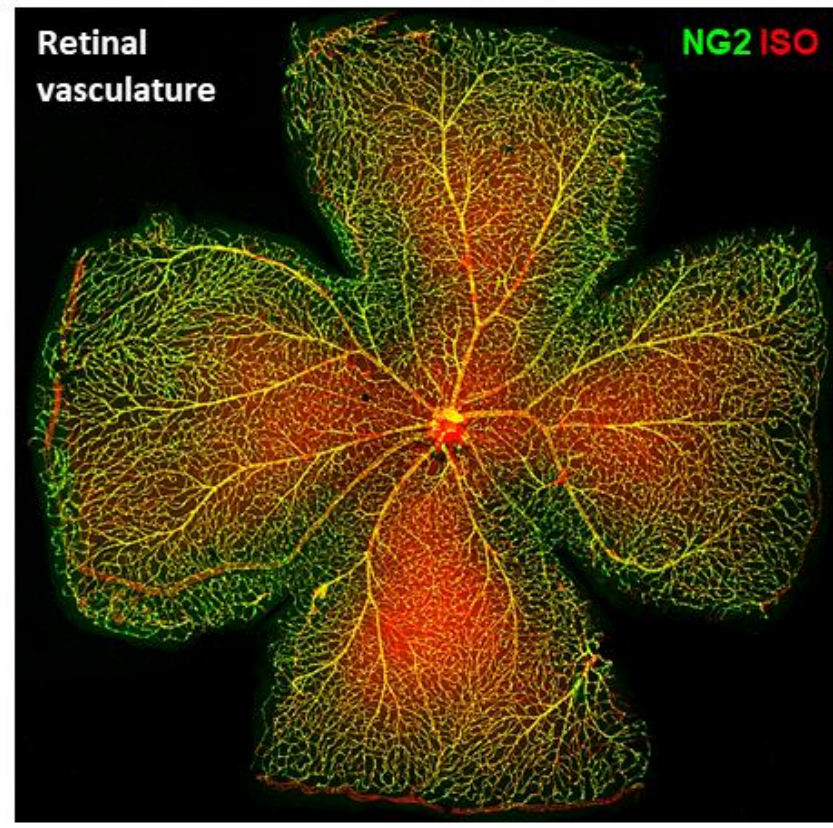

b

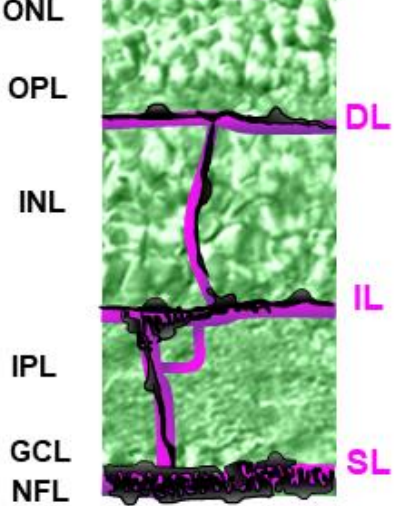

C Tight junction
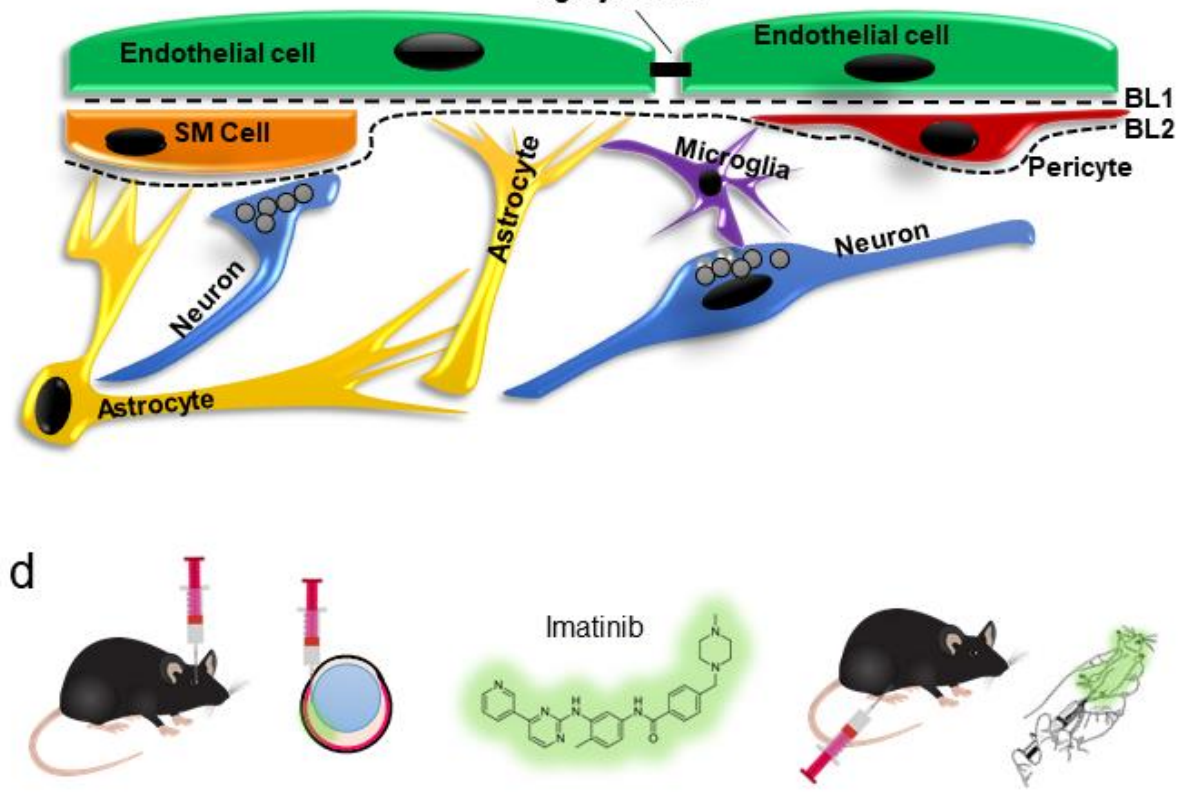

Figure 1. The essential role of pericytes in the retina and in the neural tissue. (a) Pericytes (NG2 green) and retinal vasculature in the mouse retina (IL, Isolectin GS-IB4 - red). (b) Layers of the retinal vasculature: superficial, intermediate, deep: SL, IL, DL (c) Location of pericytes in the neurovascular unit (NVU) Imatinib treatment schemes. (d) Local (intravitreal -IV, left) and global (intraperitoneal $\mathrm{IP}$, right) treatment with imatinib.

\section{Results}

The retinal vasculature supplies the retina with an adequate amount of oxygen and metabolites (Fig. 1a, b), thereby proving essential for retinal survival. We utilized two treatment options, systemic - intraperitoneal injections and local -intravitreal injections of Imatinib to evaluate the effect of PDGF-inhibition (Fig. 1d). According to our hypothesis, imatinib treatment acts on pericytes in the adult retina and thus a decrease in pericyte number accompanied by a decrease of vascular 
density and interference in the neurovascular unit (NVU; Fig. 1c) function is expected that leads to various forms of retinal pathologies.
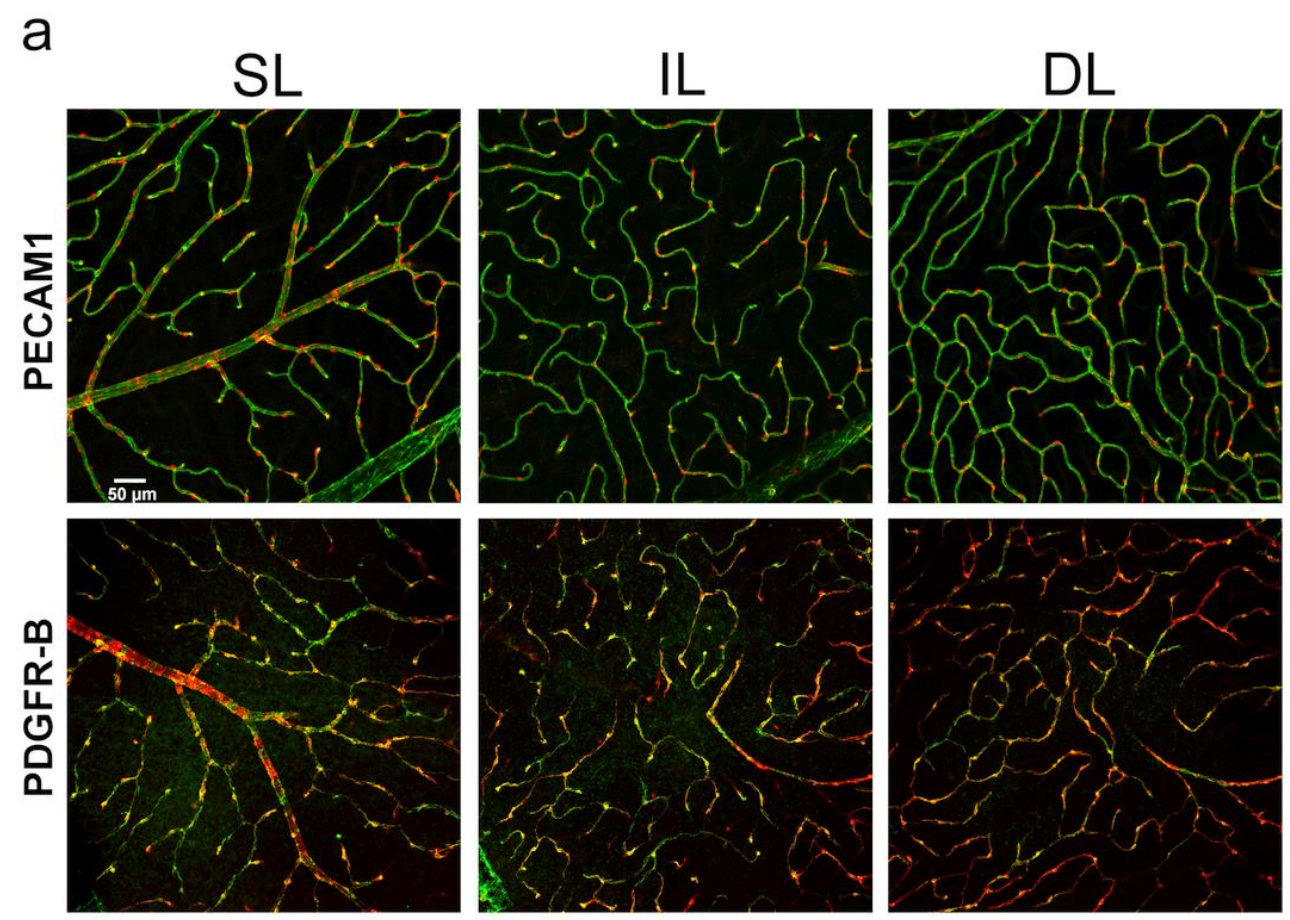

$\mathrm{b}$
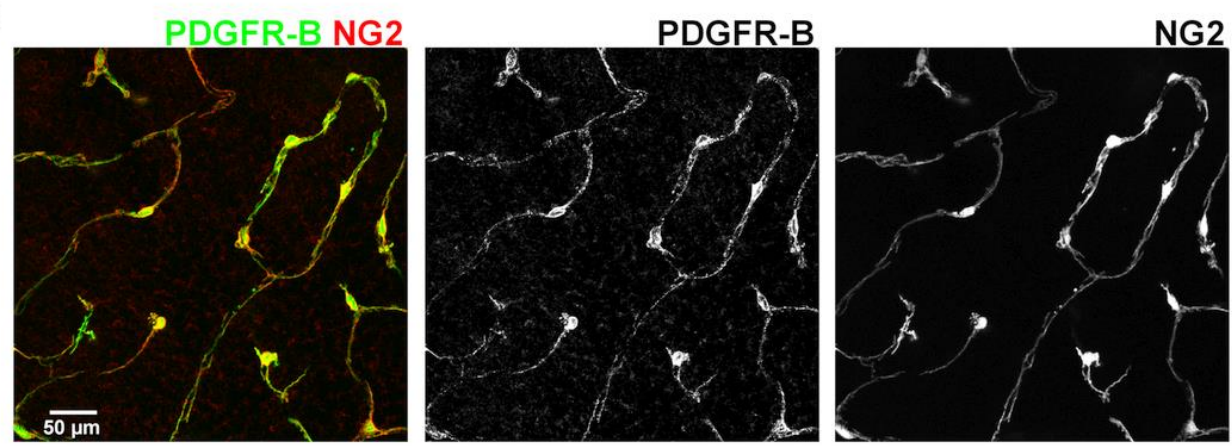

Figure 2. Laminar expression pattern of PECAM1 (CD31), PDGFR- $\beta$ and NG2 in the mouse retina shows colocalization of PDGFR- $\beta$ and NG2. Confocal images of NG2-DsRed (red) and PECAM1/PDGFR- $\beta$ (green) labeling (area 637x637 um) in mouse retinas from each (superficial-, intermediate-, deep-: SL, IL, DL) layer. PECAM1 labels endothelial cells, while PDGFR- $\beta$ is expressed by NG2+ pericytes and smooth muscle cells. It recruits vascularization and stabilizes the mature vasculature. Control. Scale: $50 \mu \mathrm{m}$.

\subsection{PDGFR- $\beta$ expression in the mouse retina shows colocalization with NG2}

We performed PDGFR- $\beta$ and PECAM1 immunohistochemical fluorescent labeling ( $\mathrm{n}=3$ retinas) in control retinas to show their colocalization with NG2. PCs express PDGFR $\beta$ and NG2 in a colocalized manner. PECAM1 is expressed by endothelial cells similar to normal adult retinas. We also tested our sham-control samples for any evident errors caused by PBS injection. We found no vascular aberrations in our sham control samples (Fig. 2).

\subsection{Imatinib decreases blood vessel coverage}

First, we used albumin labeling to evaluate the effect of Imatinib on the vascular densities in each retinal layer. It was not evident if Imatinib induced any change in the blood vessel coverage 
when albumin labeled confocal images were compared. To also obtain a quantitative comparison we performed a measurement of areal coverage of blood vessels in all layers of sham controls, IV and IP treated mouse retinas. Based on this comparison our data suggest that Imatinib treatment induced a decrease in BV coverage in all layers for both treatment modalities. This decrement was evident and statistically significant in the deep layer and the intermediate layer of the IP injected animals $(n=4$ mice for C, 3 IP, 3 IV; ANOVA; Tukey's post hoc; p<0.05) (Fig. 3a, d, e).
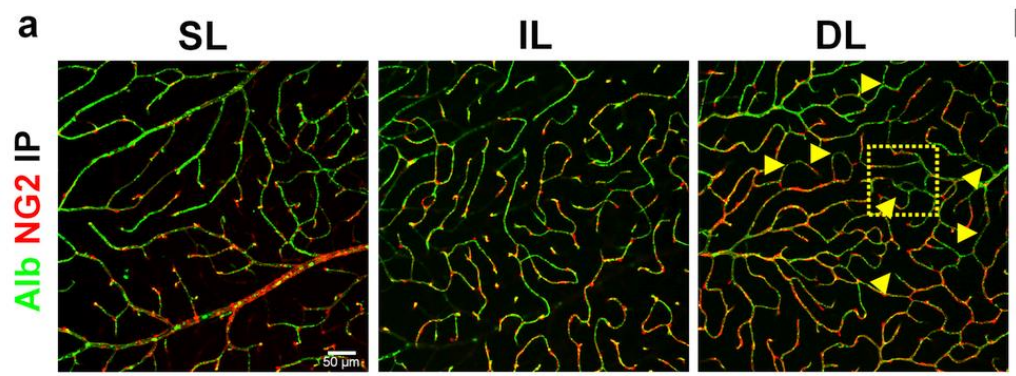

\section{b Magnified}
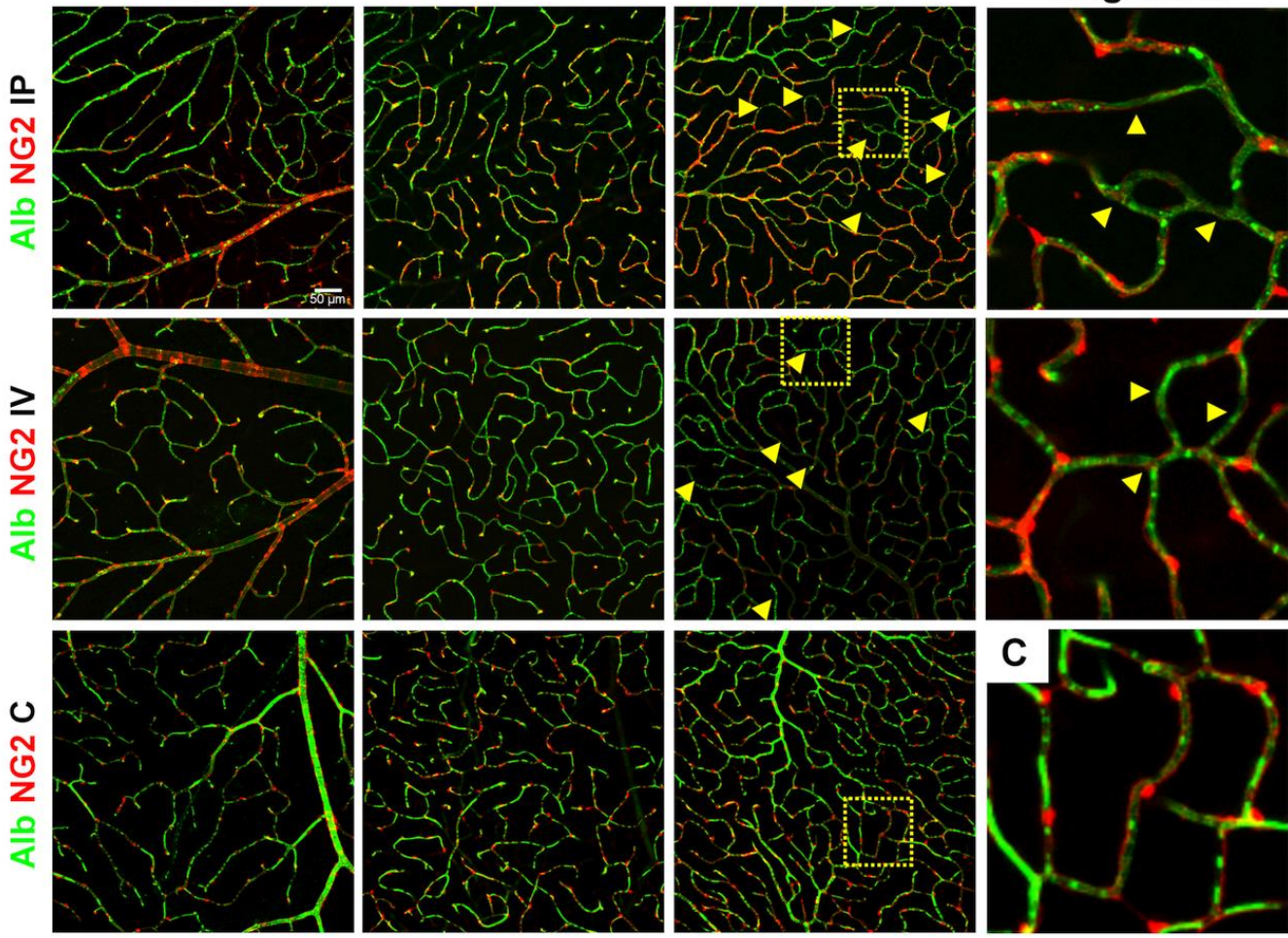

d Measurement of BV area (C)

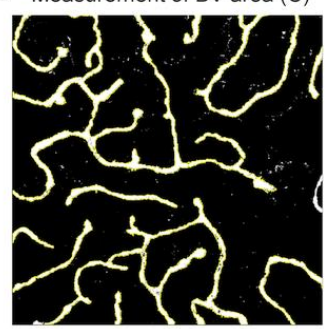

e 20

Area of BVs

$\left(300 \mu \mathrm{m}^{2}\right)$

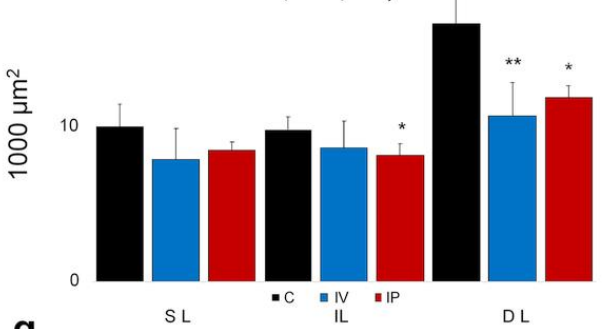

g
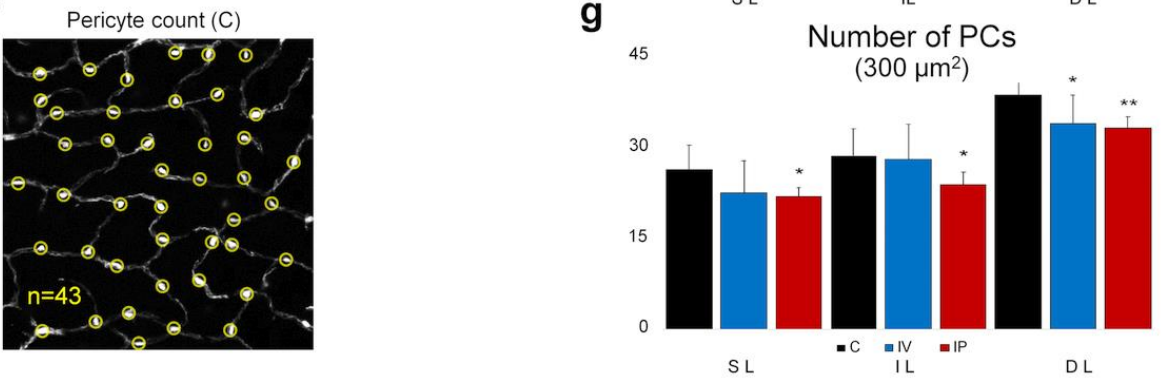

DL

Figure 3. Reduction of pericyte count and blood vessel areal densities following Imatinib treatment (a) Confocal images of NG2-DsRed (red) and Albumin (green) labeling (area 637x637 um) in mouse retinas from each layer (superficial-, intermediate-, deep-: SL, IL, DL). Measurements were made from these original images, layer-by-layer, in a $300 * 300 \mu \mathrm{m}$ area in sham-injected (C), Intravitreally injected ( $2 \mu \mathrm{l}, 25 \mathrm{mM}$ Imatinib, IV) and intraperitoneally injected $(100 \mathrm{mg} / \mathrm{kg} / \mathrm{d}, 2$ days Imatinib, IP) retinas. We found no obvious sign of any extravascular albumin label in our Imatinib treated 
specimen (nor our controls), suggesting that the brain-retina barrier (BRB) remained intact in retinas of these mice. $n=10$ (C 4, IV 3, IP 3). PC loss is indicated with yellow arrows. (b) magnified image from IP and IV showing PC dropout (arrows) and surrounding PCs with extended processes. (c) We could not see, or only sporadically see the same PC loss in sham controls. An example for (d) measurement BV areas and (f) counting PCs. (e) BV areas and PC numbers (g) are decreased in the IL and DL and suggested in the SL and in the IL of IV animals. Scale: $50 \mu \mathrm{m}$. Magnified cutout origins labeled with yellow squares in (a).

\subsection{Imatinib treatment decreases the number of pericytes}

Pericytes are essential for blood-vessel formation [4] therefore we tested if the above-observed decrease of BV areas (Fig. 3e) was accompanied by a corresponding change in the number of PCs (Fig. 3f) following Imatinib treatment. Our data suggest that both treatment modalities resulted in a decrease in PC coverage in all three vascular layers. This decrease was pronounced in all three layers of the IP injected mice and in the deep layer in the IV treated animals $(\mathrm{p}<0.5)$ (Fig. 3g). This means a $14.7,17.07 \%$ decrease in the SL, in the IV and IP; a 1.83 and $16.59 \%$ decrease in the IL and 12.15 and $14,01 \%$ decrease in the IV, IP in the DL of treated animals (Table 1). This thus means that Imatinib treatment results in a decrease of the pericyte numbers in all layers and for both treatments. In addition, it also suggests a milder effect of local treatment in all layers.

Table 1. Layer specific PC counts in the experimental specimens

PC numbers

\begin{tabular}{|c|c|c|c|c|c|c|c|c|c|}
\hline \multicolumn{2}{|c|}{ Treatment } & \multicolumn{2}{|l|}{ Position } & \multicolumn{2}{|l|}{ Mean } & \multicolumn{2}{|l|}{ SD } & \multicolumn{2}{|l|}{$\mathbf{N}$} \\
\hline & \multirow[t]{3}{*}{ C } & DL & & 38.375 & & 3.114 & & 8 & \\
\hline & & IL & & 28.375 & & 4.502 & & 8 & \\
\hline & & SL & & 26.125 & & 3.980 & & 8 & \\
\hline & \multirow[t]{3}{*}{ IP } & $\mathrm{DL}$ & & 33.000 & & 1.732 & & 3 & \\
\hline & & IL & & 23.667 & & 2.082 & & 3 & \\
\hline & & SL & & 21.667 & & 1.528 & & 3 & \\
\hline & \multirow[t]{3}{*}{ IV } & DL & & 33.714 & & 4.572 & & 7 & \\
\hline & & IL & & 27.857 & & 5.669 & & 7 & \\
\hline & & SL & & 22.286 & & 5.251 & & 7 & \\
\hline & C SL & IV SL & IP SL & C IL & IV IL & IP IL & C DL & IV DL & IP DL \\
\hline Avg. PC nr. & 26.13 & 22.29 & 21.67 & 28.38 & 27.86 & 23.67 & 38.38 & 33.71 & 33.00 \\
\hline SD & 3.98 & 5.25 & 1.53 & 4.50 & 5.67 & 2.08 & 3.11 & 4.57 & 1.73 \\
\hline$\%$ & 100.00 & 85.30 & 82.93 & 100.00 & 98.17 & 83.41 & 100.00 & 87.85 & 85.99 \\
\hline Change (\%) & & -14.70 & -17.07 & & -1.83 & -16.59 & & -12.15 & -14.01 \\
\hline
\end{tabular}

${ }^{*} \mathrm{C}$ : Sham-treated control, IP: intraperitoneal- and IV: intravitreal imatinib treated

To test whether there was a significant correlation between the change in pericyte number and the decrease in capillary surface area, using R (R Core Team 2013) we performed a Spearman's rank correlation test $(\mathrm{p}<0.01$, rho $=0.7743385)$ and also fitted a Generalized Linear Model to our dataset, setting the capillary layer and the treatment type as factors (Pericyte number - Area: $\mathrm{p}=0.039199)$. The results suggest a strong correlation between the pericyte number and capillary area.

\subsection{Imatinib treatment does not change the length of capillaries but PC coverage and capillary diameter}

Having proved that Imatinib treatment caused PC loss (Fig. 3g), the next step was to determine whether the loss of PCs had an effect on BVs. However, the total lengths in all layers remained unchanged in any of the treatment modalities (Fig. 4a, b). Taking into account the PC-loss in both the 
IP and IV injected mice it was not surprising that the BV length vs. PC number ratio positively changed after both of the treatments in the IL and DL and in the SL of the IV injected animals. The same effect was suggested in the SL of the IP injected mice, but without significant change (Fig. 4c). However, since BV area coverage decreased, while the capillary length remained constant, we had to test where the decrease in the area comes from. We randomly tested capillary diameters in our dataset to show whether imatinib injection has an effect. In the case of both types of imatinib treatment modalities capillary diameters decreased significantly in the IL and DL measured next to PC somas (Fig. 4d, e), making this decrease in diameter responsible for the decrease in BV area coverage.

a

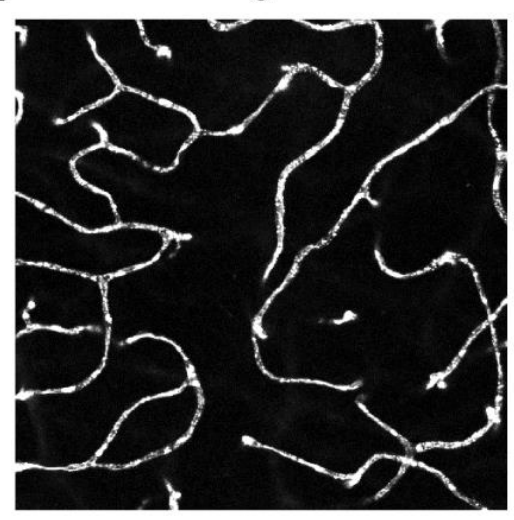

\section{d}

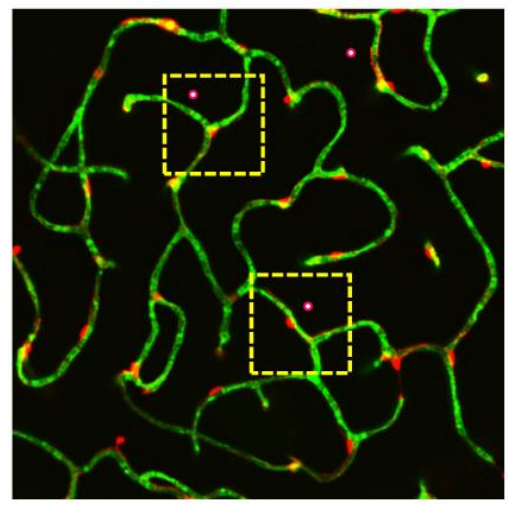

Length measurement
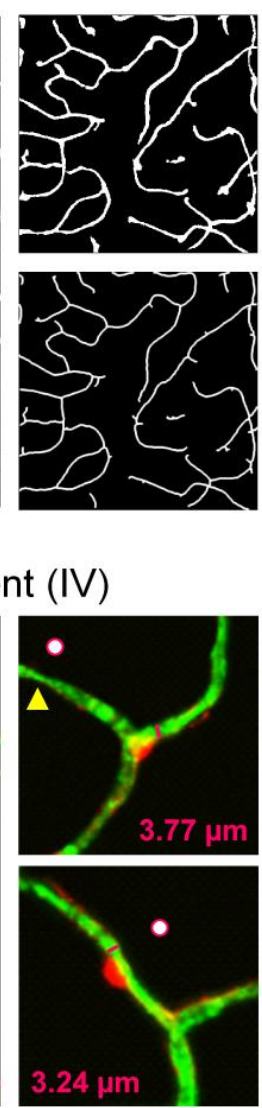

b

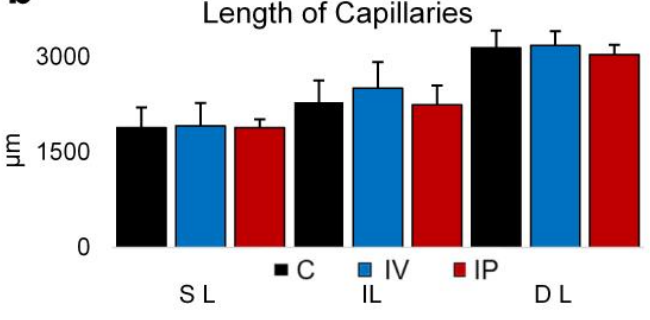

C

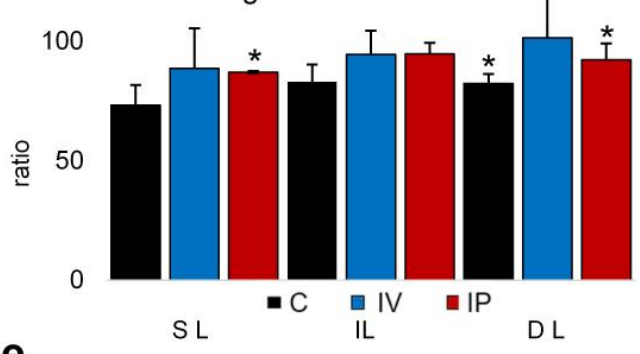

BV length / PC number ratio *

e

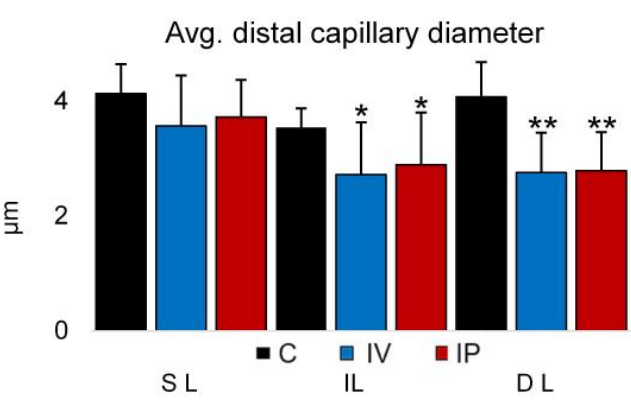

Figure 4. Reduction of pericyte number is not accompanied by BV length shrinkage following Imatinib treatment (a) Illustrated step-by-step method for BV length measurement: normalized BW image of albumin IHC-labels merged from $n=5$ optical slides (top); binarized image (right top); the skeleton of BVs used to measure length (right bottom). (b) Comparison of blood vessel lengths in retinal layers in Control (C), Intravitreally injected $(2 \mu \mathrm{l}, 25 \mathrm{mM}$ Imatinib, IV) and intraperitoneally injected $(100 \mathrm{mg} / \mathrm{kg} / \mathrm{d}$, 2 days Imatinib, IP) retinas. (c) Comparison of BV length / PC number ratios on the same dataset suggests that PCs fill out the gaps after PC loss and protect the underlying vasculature. Measurements on capillary diameters (d) show a reduction in the IL and DL (e). Measurements were made layer-by-layer in a $0.3^{*} 0.3 \mathrm{~mm}$ area from a mid-central region. C, IV, IP $\mathrm{n}=4,4$, 3. Error bars $\pm \mathrm{SD} .{ }^{*} \mathrm{P}<0.05 ;{ }^{* *} \mathrm{P}<0.01$.

\subsection{Imatinib did not induce BRB impairment.}

In the albumin labeled samples, no albumin leakage into the extracellular space was detected, which would have indicated a BRB impairment (Fig. 3a). This thus confirms that the BRB was intact in both sham control and Imatinib treated animals and further suggests that Imatinib treatment caused milder degeneration, without BRB breach in the retinal tissue as generally observed in other degenerative processes [32]. 
Notably, IP treated mice had an average of $11.15 \%$ decrease $(p=0.065)$ in body weight 7 days after the first injection, suggesting a global negative effect of Imatinib treatment. Nonetheless, we could not detect any characteristic change in the IV and the Control animal's weight.

\section{Discussion}

Several conditions and diseases, such as diabetes mellitus, hypertension, Age-related macular degeneration (AMD) are associated with negative effects on the development and structure of the retinal vasculature [33,34]. Hypertension, for example, causes an increased contractile potential of the vessel walls and later PC loss [35]. Hyperglycemia in diabetes mellitus can also lead to microvascular diseases, including diabetic retinopathy [36].

It remains a great challenge to define the key vascular components of diabetic retinopathy, however, our present knowledge and recent discoveries in this field showed that PCs are crucial in BV formation and maintaining its structure [36]. Diabetic retinopathy is characterized by several morphological changes, including loss of pericytes, thickening of the basement membrane, increased vascular permeability, vascular occlusion, and microaneurysm. Therefore, it can be concluded, that PC depletion is one of the major hallmarks of Diabetic retinopathy [15]. Although, intraocular anti-VEGF injection and laser treatments can help regress these abnormal new vessels but they both can be detrimental or inadequate options for long term treatment [37]. Imatinib, as a potent PDGFR-, tyrosine kinase- and KIT inhibitor has been successfully used in treating cancer, and thus could provide an alternative treatment strategy in neurovascular diseases. It has also been shown previously that using Imatinib as a PDGFR inhibitor during retinal development after OIR (oxygen-induced retinal) neovascularization successfully ameliorated retinal degeneration in the developing retina [24]. In this study, we found that the number of PCs were depleted in all three layers of the retinal BVs in the healthy, adult retina (in a fixed area, not along BVs). The deep layer was the most affected, out of the three layers, in both utilized treatment options. In addition, the decreased PC number was accompanied by a reduced capillary area and this correlation first suggested a strong association or even a causative link between these Imatinib induced effects. However, we found no observable Imatinib induced a change in the length of blood vessels. As PCs line up along capillaries their numbers are expected to follow the length rather than the summated area of the capillary system. Our observations therefore suggested a PC rearrangement. This hypothesis was supported by the Imatinib induced increase of the BV area/ PC number ratio in both treatment modalities. This finding suggests that each retained PC covers a lengthened BV section to compensate for the Imatinib induced PC loss. While BV areas actively change in live animals it is uncertain if the observed BV area reduction in the post-fixed state represents a corresponding BV area decrease in the live animal. Assuming that the constricted state is preserved in the fixed tissue the detected decrease in BV areas suggests that PCs constrict after imatinib treatment. Besides the above Imatinib induced changes layer specific differences in PC loss was also observed (Fig 4; Table 1), which can be explained by the efficacy of local versus systemic treatment modalities. The SL and DL are similarly affected, while the IL is less affected by IV treatment. This may suggest that the DL is more sensitive to Imatinib treatment, despite the lover local dose in IV, whereas the highest dose occurs at the level of the nearest vascular layer, the SL.

Inhibition of PDGFs/PDGFRs might be a promising therapy to suppress pathological angiogenesis. To test for Imatinib induced side effects, we measured the bodyweight of the mice before and after the treatment and labeled the retinas with albumin, to detect any BRB breakdown. Our results show no hemorrhage and BRB breakdown after Imatinib treatment either in local- or in systemic treatment tests (Fig3). This evidence suggests that short exposure to Imatinib $\left(1^{*} 2 \mathrm{ul}\right.$ (8ug/ul) IV; $2^{*} 100 \mathrm{mg} / \mathrm{kg} /$ day IP) already has an effect on PC density and vascular area, but not on the length and $B R B$ integrity. This observation is consistent with other studies suggesting that the PDGF-B/PDGFR- $\beta$ signaling is not required to maintain EC-pericyte interaction for BRB integrity during adulthood, although it is indispensable in formation and maturation of BRB through active recruitment of pericytes onto the growing retinal vessels and also could be related to the suggested BRB protective effect of Imatinib [17]. A weight drop occurred in the IP injected, but not in the IV 
injected animals after treatment, suggesting an adverse systemic effect of Imatinib that can be avoided with local administration. These findings, therefore, suggest that local Imatinib administration could be considered as an additional treatment modality. On the other hand, the significant weight-loss occurred after the systemic treatment points towards negative side effects of the systemic Imatinib administration.

In human chemotherapy, Imatinib dose varies between 100-600 mg daily [38]. The highest clinical dose equals $8-12 \mathrm{mg} / \mathrm{kg} /$ day (depending on individual weight), whereas our experimental animals were treated with $100 \mathrm{mg} / \mathrm{kg} /$ day for only 2 days. Interestingly, however, this applied much higher dose was not disruptive on the BRB integrity but decreased the PC numbers in all layers. This observation seemingly contradicts a previous study of Armulik et al. [17], where no change was detected in the BV coverage and PC numbers after Imatinib treatment. However, in that study, a shorter survival-time $(48 \mathrm{~h})$ was applied after the first treatment, whereas we waited7 days to provide enough time to any possible rearrangement. This difference in the applied methodologies can easily explain the observed discrepancies. Additionally, local Imatinib treatment may also have a somewhat different effect on retinal BV formation and maintenance.

If used for a longer period, Imatinib could cause retinal pathologies in chemotherapy [30, 31, 39]. This long term Imatinib effect on vasculature may be explained by the loss of the PC dynamic interactions thus the ability to sustain retinal metabolic needs in some conditions [6].

Also, undeniably, Imatinib treatment is not specific for PC elimination, since it is not exclusively specific for PDGFR-B, it could serve as a good model for pericyte dropout, having a role in multiple diseases. To focus its effect and to avoid possible side effects, our results suggest a local treatment on BVs if possible. Since Imatinib has been approved for medical use, there are fewer obstacles ahead to benefit from its effect.

Finally, as we have recently shown, PCs form an essential 3D mosaic across the retina to provide a propagating contraction / dilation of capillaries. The signal for this propagation travels across gap junctions between neighboring PCs, thereby precisely supporting metabolic needs in the nervous tissue [22]. Any loss in this 3D PC structure contributes to the loss of this essential function. We theorize that the PC mosaic can adapt to such changes to restore the function by filling out the gaps along the vasculature primarily resulted from the loss of some PCs. The given PC-loss, without a negative change in BV length, suggests that PCs may extend their processes and even move to rebuild the mosaic in order to protect $\mathrm{BV}$ s from degeneration. The decrease in capillary areas may come from either a decrease in their length or a decrease in diameter. We found that the diameters indeed shrunk after treatments. Although this shows a controversy with other findings [17], it could be explained by the longer recovery time (7 days). After our treatments the stress that might be still present caused by PC-mosaic reorganization. Although this theory needs to be further tested, it also serves as a direct explanation and a future path for pericyte research.

\section{Materials and Methods}

\subsection{Animals \& Imatinib treatment}

Animal handling, housing, and experimental procedures were reviewed and approved by the ethical committee of the University of Pécs (BA02/2000-6/2006; BA/35/51-42/2016) and in compliance with protocols approved by the Institutional Animal Care and Use Committee of Weill Cornell Medicine. All animals were treated in accordance with the ARVO Statement for the Use of Animals in Ophthalmic and Vision Research and with the National Institutes of Health Guide for the care and use of laboratory animals. All efforts were made to minimize pain and discomfort during the experiments and all procedures were done by obeying the 3R law.

We used multiple routes of Imatinib administration to evaluate the effects of global, and local use of Imatinib. Two mouse lines: C57BL/6 mice (The Jackson Laboratory, stock \#000664, RRID:IMSR_JAX:000664) and NG2-DsRed mice (The Jackson Laboratory, $\operatorname{Tg}$ (Cspg4-DsRed.T1)1Akik/J, stock \#008241, RRID:IMSR_JAX:008241) were used in this study. 
Animals were injected either with Imatinib (Methanesulfonate Salt, LC Laboratories, Cat. I-5508) or filtered phosphate-buffered saline (PBS; $0.1 \mathrm{M}, \mathrm{pH}=7.3$ ) as follows:

A: Imatinib intraperitoneal injection (IP) $100 \mathrm{mg} / \mathrm{kg} /$ day Imatinib (dosage according to Raimondi 2014) in filtered PBS for 2 days, on $n=4$ mice.

B: Imatinib intravitreal injection (IV) $2 \mathrm{ul}(8 \mathrm{ug} / \mathrm{ul})$ in filtered PBS on $\mathrm{n}=3$ mice.

C: A/B equal volume injected from filtered PBS both IP and IV on $n=4$ mice; used as a sham control.

\subsection{Immunohistochemistry}

Eyecups of mice were fixed with $4 \%$ carbodiimide and $0.25 \%$ PFA in $0.1 \mathrm{M}$ phosphate saline (PBS, $\mathrm{pH}$ 7.3) for $15 \mathrm{~min}$ at room temperature. After fixation, the samples were washed in PBS, and the retinas were dissected. Retinal whole mounts were blocked for 8-14 h in CTA (PBS solution containing 5\% Chemiblocker (membrane-blocking agent, Millipore), 0.5\% Triton X-100, and 0.05\% sodium azide, Sigma). Primary antibodies were diluted in the same solution and applied for $72 \mathrm{~h}$, followed by incubation for $48 \mathrm{~h}$ in the appropriate secondary antibody, conjugated to Alexa-488 (1:1000; green fluorescence, Invitrogen), Alexa-568 (1:1000; red fluorescence, Invitrogen), Alexa-633 (1:500; far-red fluorescence, Invitrogen). In multi labeling experiments, whole mounts were incubated in a mixture of primary antibodies, followed by a mixture of secondary antibodies. All steps were performed at room temperature. After staining, the retinal pieces were flat-mounted on a slide, ganglion cell layer up, and coverslipped using Vectashield mounting medium (H-1000, Vector Laboratories). The coverslip was sealed with nail polish using small pieces of a broken glass coverslip (number 1 size) as spacers. The primary antibodies used in this study were the following: rabbit anti-NG2 coupled to Cy3 fluorescent label (NG2, 1:500, EMD Millipore, AB5320C3), goat anti-mouse albumin (albumin, 1:1000, Bethyl Laboratories, A90-234A), goat anti-mouse PECAM1 (alternatively CD31; 1:5000, R\&D Systems, AF3628) goat anti-mouse PDGFR- $\beta$ (1:5000, R\&D Systems, AF1042). Blood vessels were visualized by Isolectin coupled to Alexa-488 fluorescent label (1:500, Invitrogen, I21411) to show the whole retinal vasculature with secondary antibodies. Retinal samples were imaged under a Nikon Eclipse Ti-U confocal microscope. For pericyte-evaluation retinas were imaged with 20x objective and areas of $300 \mathrm{x}$ $300 \mu^{2}$ were selected for measurements (5 IP animals, 10 areas; 3 IV animals, 6 areas; 5 control animals, 8 areas). The samples were imaged under identical acquisition conditions, including laser intensity, photomultiplier amplification, and Z-stack step size.

\subsection{Image analysis}

All images were processed and analyzed using FIJI software (ImageJ, NIH; [40]). For measurements, we used areas of $300 \times 300 \mu \mathrm{m} 2$, merged (from 5-8 optical slide) images. Blood vessels, labeled by Albumin, were traced with thresholding in FIJI and their total area was measured. NG2-positive pericyte numbers were counted along capillaries, taking into account the local BV diameter and cell morphology, using the cell counter plugin in FIJI. In total, we evaluated 1565 individual pericytes from all sample sets.

Albumin labeling was also used to evaluate BV length. After initial thresholding and global processing, images were converted to binary black and white. We used the dilate and erode binary function subsequently to smooth local unevenness at BV edges. Skeletonized BVs were measured using the analyze skeleton function in FIJI. Results were transferred to Excel for further processing.

Distal capillary diameter evaluation was made on composite images. First, a custom made ImageJ script was used to generate random points on the images. We measured the diameter with the FIJI line tool on the nearest capillary from this point, next to the PC soma (Fig4d).

\subsection{Statistical analysis}

For statistical analysis, Excel (Microsoft Corp., Redmond, USA), JASP (JASP Team, 2019 JASP, V 0.11.1), and $R$ (R Core Team 2013) were used. An independent t-test was used to determine the $\mathrm{BV} / \mathrm{PC}$ number ratio significance. For multiple comparisons, analysis of variance (ANOVA) with 
post-hoc Tukey's test or repeated-measures ANOVA was used with Pearson's correlation matrix. The number of samples (n) indicates the number of mice in the group. The data is presented as mean \pm SD unless otherwise indicated.

Author Contributions: conceptualization, T.KÖ. and B.S.; methodology, T.KÖ., E.I.; validation, T.KÖ., B.S.; formal analysis, T.KÖ., G.S., A.T.; investigation, T.KÖ.; resources, B.S.; data curation, T.KÖ., G.S., A.T.; writing-original draft preparation, T.KÖ.; writing-review and editing, B.S., B.V., E.I., A.T., G.S., T.KÖ.; visualization, T.KÖ.; supervision, B.S.; funding acquisition, B.S., B.V.

Funding: This research was supported by NIH grants R01-EY026576 and R01-EY029796 (B.S.). This study was also supported by the Hungarian Brain Research Program 2 (2017-1.2.1.-NKP-2017) to B.V., by NKFI (OTKA NN128293) to B.V., by the European Union and the State of Hungary, co-financed by the European Social Fund in the framework of TAMOP-4.2.4.A/ 2-11/1-2012-0001 National Excellence Program (B.V.) and by the ÚNKP-19-19-2-I-PTE-96 New National Excellence Program of the Ministry for Innovation and Technology (G.S.).

Acknowledgments: We thank E. KÖ. for the help.

Conflicts of Interest: The authors declare no conflict of interest.

\section{Abbreviations}

a-SMA Alpha-smooth muscle actin

ALS Amyotrophic lateral sclerosis

AMD Age-related macular degeneration

BBB Blood-brain barrier

BRB Blood-retina barrier

BV Blood Vessel

BW Black and white

C Control

Ch Choroid

CNS Central nervous system

Cspg4 Chondroitin sulfate proteoglycan 4

DL Deep Layer

EC Endothelial cell

GCL Ganglion cell layer

GS-IB4 Griffonia simplicifolia - IB4

IHC Immunohistochemistry

IL Intermediate Layer

INL Inner nuclear layer

IP Intraperitoneal

IPL Inner plexiform layer

IV Intravitreal

MS Microsoft

NFL Nerve fiber layer

NG2 Neuron-glial antigen 2

NVU Neurovascular Unit 


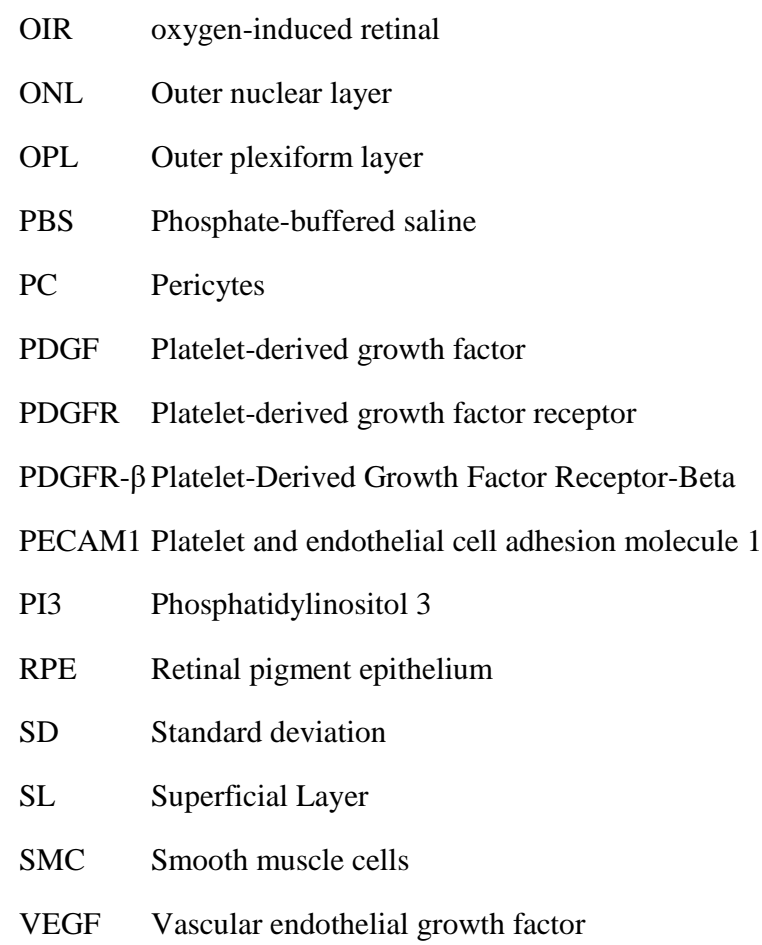

\section{References}

1. Stapor, P. C., Sweat, R. S., Dashti, D. C., Betancourt, A. M., \& Murfee, W. L. Pericyte Dynamics during Angiogenesis: New Insights from New Identities. Journal of Vascular Research, 2014, 51(3), 163-174. doi:10.1159/000362276

2. Hye-Ryong Shim, A., Liu, H., Focia, P. J., Chen, X., Lin, P. C., \& He, X. Structures of a platelet-derived growth factor/propeptide complex and a platelet-derived growth factor/receptor complex. Proceedings of the National Academy of Sciences, 2010, 107(25), 11307-11312. doi:10.1073/pnas.1000806107

3. Hill, R. A., Tong, L., Yuan, P., Murikinati, S., Gupta, S., \& Grutzendler, J. Regional Blood Flow in the Normal and Ischemic Brain Is Controlled by Arteriolar Smooth Muscle Cell Contractility and Not by Capillary Pericytes. Neuron, 2015, 87(1), 95-110. doi:10.1016/j.neuron.2015.06.001

4. Sweeney, M. D., Ayyadurai, S., \& Zlokovic, B. V. Pericytes of the neurovascular unit: key functions and signaling pathways. Nature Neuroscience, 2016, 19(6), 771-783. doi:10.1038/nn.4288

5. Hall, C.N., Reynell, C., Gesslein, B., Hamilton, N.B., Mishra, A., Sutherland, B.A., O'Farrell, F.M., Buchan, A.M., Lauritzen, M., Attwell, D. Capillary pericytes regulate cerebral blood flow in health and disease. Nature, 2014, 508(7494), 55-60. doi:10.1038/nature13165

6. Ivanova, E., Kovacs-Oller, T., \& Sagdullaev, B. T. Vascular Pericyte Impairment and Connexin43 Gap Junction Deficit Contribute to Vasomotor Decline in Diabetic Retinopathy. The Journal of Neuroscience, 2017, 37(32), 7580-7594. doi:10.1523/jneurosci.0187-17.2017

7. Ivanova, E., Kovacs-Oller, T., \& Sagdullaev, B. T. Domain-specific distribution of gap junctions defines cellular coupling to establish a vascular relay in the retina. Journal of Comparative Neurology, 2019 doi:10.1002/cne.24699

8. Khennouf, L., Gesslein, B., Brazhe, A., Octeau, J. C., Kutuzov, N., Khakh, B. S., \& Lauritzen, M. Active role of capillary pericytes during stimulation-induced activity and spreading depolarization. Brain, 2018 141(7), 2032-2046. doi:10.1093/brain/awy143

9. Alarcon-Martinez, L., Yilmaz-Ozcan, S., Yemisci, M., Schallek, J., Kılıc, K., Can, A., Di Polo, A., Dalkara, T. Capillary pericytes express $\alpha$-smooth muscle actin, which requires prevention of filamentous-actin depolymerization for detection. eLife, 2018 7, doi:10.7554/elife.34861

10. Pfister, F., Lin, J., \& Hammes, H.-P. Pericyte Loss in the Diabetic Retina. Frontiers in Diabetes, 2009, 61-78. doi:10.1159/000262662

11. Beltramo, E. \& Porta, M. Pericyte Loss in Diabetic Retinopathy: Mechanisms and Consequences. Current medicinal chemistry, 2013 doi:20. 10.2174/09298673113209990022. 
12. Bell, R. D., Winkler, E. A., Sagare, A. P., Singh, I., LaRue, B., Deane, R., Zlokovic, B. V. Pericytes control key neurovascular functions and neuronal phenotype in the adult brain and during brain aging. Neuron, 2010, 68(3):409-27, doi: 0.1016/j.neuron.2010.09.043.

13. Lindahl, P.Pericyte Loss and Microaneurysm Formation in PDGF-B-Deficient Mice. Science, 1997, 277(5323), 242-245. doi:10.1126/science.277.5323.242

14. Enge, M. Endothelium-specific platelet-derived growth factor-B ablation mimics diabetic retinopathy. The EMBO Journal, 2002, 21(16), 4307-4316. doi:10.1093/emboj/cdf418

15. Lee, S. E., \& Park, Y. S. The role of bacterial cellulose in artificial blood vessels. Molecular E Cellular Toxicology, 2017, 13(3), 257-261. doi:10.1007/s13273-017-0028-3

16. Santos, G. S. P., Prazeres, P. H. D. M., Mintz, A., Birbrair, A. Role of pericytes in the retina. Eye, 2018, 32(3), 1476-5454, doi: https://doi.org/10.1038/eye.2017.220

17. Armulik, A., Genové, G., Mäe, M., Nisancioglu, M. H., Wallgard, E., Niaudet, C., He, L., Norlin, J., Lindblom, P., Strittmatter, K., Johansson, B. R., Betsholtz, C. Pericytes regulate the blood-brain barrier. Nature, 2010 468(7323), 557-561. doi:10.1038/nature09522

18. Daneman, R., Zhou, L., Kebede, A. A., \& Barres, B. A. Pericytes are required for blood-brain barrier integrity during embryogenesis. Nature, 2010, 468(7323), 562-566. doi:10.1038/nature09513

19. Berthiaume, A.-A., Grant, R. I., McDowell, K. P., Underly, R. G., Hartmann, D. A., Levy, M., Bhat, NR. Shih, A. Y. Dynamic Remodeling of Pericytes In Vivo Maintains Capillary Coverage in the Adult Mouse Brain. Cell Reports, 2018 22(1), 8-16. doi:10.1016/j.celrep.2017.12.016

20. Park D. Y., Lee, J., Kim, J., Kim, K., Hong, S., Han, S., Kubota, Y., Augustin, H. G., Ding, L., Kim, J., W., Kim, H., He, Y., Adams, R. H. \& Koh, G. Y.. Plastic roles of pericytes in the blood-retinal barrier. Nature Communications, 2017, 8, 15296, doi:10.1038/ncomms15296

21. Sennino, B., Falcon, B. L., McCauley, D., Le, T., McCauley, T., Kurz, J. C., Haskell, A., Epstein, D. M., McDonald, D. M. Sequential Loss of Tumor Vessel Pericytes and Endothelial Cells after Inhibition of Platelet-Derived Growth Factor B by Selective Aptamer AX102. Cancer Research, 2007 67(15), 7358-7367. doi:10.1158/0008-5472.can-07-0293

22. Kovacs-Oller, T., Ivanova, E., Bianchimano, P., Sagdullaev, B. T. Dynamic connectivity maps of pericytes and endothelial cells mediate neurovascular coupling in health and disease. bioRxiv 830398, doi: https://doi.org/10.1101/830398 (pre-print)

23. Druker, B. J., Tamura, S., Buchdunger, E., Ohno, S., Segal, G. M., Fanning, S., Zimmermann, J. \& Lydon, N. B. (1996). Effects of a selective inhibitor of the Abl tyrosine kinase on the growth of Bcr-Abl positive cells. Nature Medicine, 2(5), 561-566. doi:10.1038/nm0596-561

24. Zhou, L., Sun, X., Huang, Z., Zhou, T., Zhu, X., Liu, Y., Wang, J., Cheng, B., Chang He, M. L., Liu, X. Imatinib Ameliorated Retinal Neovascularization by Suppressing PDGFR- $\alpha$ and PDGFR- $\beta$. Cellular Physiology and Biochemistry, 2018, 48(1), 263-273. doi:10.1159/000491726

25. Gergely, P. A., Murnyák, B., Bencze, J., Kurucz, A., Varjas, T., Gombos, K., \& Hortobágyi, T. Tyrosine Kinase Inhibitor Imatinib Mesylate Alters DMBA-Induced Early Onco/Suppressor Gene Expression with Tissue-Specificity in Mice. BioMed Research International, 2019, 1-12, doi:10.1155/2019/8670398

26. Biswas, S. K., Zhao, Y., \& Sandirasegarane, L. Imatinib induces apoptosis by inhibiting PDGF- but not insulin-induced PI 3-kinase/Akt survival signaling in RGC-5 retinal ganglion cells. Molecular vision, 2009, 15, 1599-1610.

27. Rajkumar, V. S., Shiwen, X., Bostrom, M., Leoni, P., Muddle, J., Ivarsson, M., Gerdin, B., Denton, C. P., Bou-Gharios, G., Black, C. M., Abraham, D. J. Platelet-Derived Growth Factor- $\beta$ Receptor Activation Is Essential for Fibroblast and Pericyte Recruitment during Cutaneous Wound Healing. The American Journal of Pathology, 2006, 169(6), 2254-2265. doi:10.2353/ajpath.2006.060196

28. Zhao, Y., Biswas, S. K., McNulty, P. H., Kozak, M., Jun, J. Y., \& Segar, L. PDGF-induced vascular smooth muscle cell proliferation is associated with dysregulation of insulin receptor substrates. American Journal of Physiology-Cell Physiology, 2011, 300(6), 1375-1385. doi:10.1152/ajpcell.00670.2008

29. Raimondi, C., Fantin, A., Lampropoulou, A., Denti, L., Chikh, A., Ruhrberg, C. Imatinib inhibits VEGF-independent angiogenesis by targeting neuropilin 1-dependent ABL1 activation in endothelial cells. J Exp Med, 2014, 211 (6), 1167-1183. doi: https://doi.org/10.1084/jem.201

30. Fraunfelder, F. W., Solomon, J., Druker, B. J., Esmaeli, B., \& Kuyl, J. Ocular Side-Effects Associated with Imatinib Mesylate (Gleevec $\left.{ }^{\circledR}\right)$. Journal of Ocular Pharmacology and Therapeutics, 2003, 19(4), 371-375. doi:10.1089/108076803322279426 
31. Gulati, A. \& Saif, M. Retinal Neovascularization and Hemorrhage Associated with the Use of Imatinib (Gleevec $\left.{ }^{\circledR}\right)$ in a Patient Being Treated for Gastrointestinal Stromal Tumor (GIST). Ar.iiarjournals.org., 2012 Available at: http://ar.iiarjournals.org/content/32/4/1375.long.

32. Ivanova, E., Alam, N. M., Prusky, G. T., \& Sagdullaev, B. T. Blood-retina barrier failure and vision loss in neuron-specific degeneration. JCI insight, 2019,5(8), e126747. doi:10.1172/jci.insight.126747

33. Friedman, E. Update of the vascular model of AMD. British Journal of Ophthalmology, 2004, 88(2), 161-163. doi:10.1136/bjo.2003.036277

34. Ehrlich, R., Harris, A., Kheradiya, N. S., Winston, D. M., Ciulla, T. A., \& Wirostko, B. (2008). Age-related macular degeneration and the aging eye. Clinical interventions in aging, 3(3), 473-482. doi:10.2147/cia.s2777

35. Wallow, I. H., Bindley, C. D., Reboussin, D. M., Gange, S. J., Fisher M. R., Systemic hypertension produces pericyte changes in retinal capillaries. Invest. Ophthalmol. Vis. Sci. 1993, 34(2), 420-430. doi: https://doi.org/.

36. Liu, C., Ge, H.-M., Liu, B.-H., Dong, R. Shan, K., Chen, X., Yao, M.-D., Li, X.-M., Yao, J., Zhou, R.-M., Zhang, S.-J., Jiang, Q., Zhao, C., \& Yan B. Targeting pericyte-endothelial cell crosstalk by circular RNA-cPWWP2A inhibition aggravates diabetes-induced microvascular dysfunction. Proceedings of the National Academy of Sciences, 2019, 201814874, doi:10.1073/pnas.1814874116

37. Li, S., Deng, G., Liu, J., Ma, Y., \& Lu, H. The effects of a treatment combination of anti-VEGF injections, laser coagulation and cryotherapy on patients with type 3 Coat's disease. BMC Ophthalmology, 2017, 17(1), doi:10.1186/s12886-017-0469-4

38. Medscape, Gleevec (imatinib) dosing, indications, interactions, adverse effects, and more. Available online: https://reference.medscape.com/drug/gleevec-imatinib-342239

39. Kusumi, E., Arakawa, A., Kami, M., Kato, D., Yuji, K., Kishi, Y., Murashige, N., Miyakoshi, S., Ueyama, J., Morinaga, S., Taniguchi, S., Muto, Y. Visual disturbance due to retinal edema as a complication of imatinib. Leukemia 2004, 18(6), 1138 - 1139 doi:https://doi.org/10.1038/sj.leu.2403364 DO 10.1038/sj.leu.2403364

40. Schindelin, J., Arganda-Carreras, I., Frise, E., Kaynig, V., Longair, M., Pietzsch, T., Preibisch, S., Rueden, C., Saalfeld, S., Schmid, B., Tinevez, J.-Y., White, D. J., Hartenstein, V., Eliceiri, K., Tomancak, P. \& Cardona, A. Fiji: an open-source platform for biological-image analysis. Nature Methods, 2012 9(7), 676-682. doi:10.1038/nmeth.2019 